\title{
Plasma and cerebrospinal fluid tryptophan in multiple sclerosis and degenerative diseases
}

\author{
F. MONACO, S. FUMERO,A. MONDINO, A N D R. MU T A N I \\ From the Department of Neurology, University of Sassari, and the RBM Institute of Biomedical \\ Research, Ivrea, Italy
}

SUMMARY Tryptophan and competing neutral amino acid levels were found to be diminished in the plasma of patients with multiple sclerosis and degenerative diseases, the greatest decrease being of tryptophan. Cerebrospinal fluid tryptophan was decreased in multiple sclerosis and motor neurone disease, while leucine and valine were increased. These changes might lead to decreased synthesis of brain serotonin and brain proteins. The ratio between neutral amino acids and tryptophan might be used as an ancillary test in the screening of degenerative diseases.

Tyrosine and phenylalanine share with leucine, isoleucine, and valine the same transport class across the blood-brain barrier, and all of them compete with tryptophan for cerebral uptake (Lajtha, 1974). Since an increased tyrosinephenylalanine quotient has been shown in serum of patients with multiple sclerosis (Kisser and Harrer, 1974), we determined the concentrations of both plasma and cerebrospinal fluid tryptophan and competing amino acids in a group of patients with multiple sclerosis and other degenerative diseases.

\section{Patients and methods}

Thirty-two patients of both sexes, aged 22-47 years, were included in the study, provided that they presented at least five of the following signs and symptoms: eye squint with diplopia, cerebellar ataxia or dysmetria or both, dysarthria, pyramidal tract signs with loss of abdominal reflexes, temporal atrophy of the optic disc, impairment of micturition. In 21 patients the diagnosis was confirmed by a CSF gammaglobulin electrophoretic pattern. At least three neurologists agreed on the diagnosis in each case. In 19 cases the disease was at its onset; while in the remainder the history was of chronic illness. None of them was on therapy at the time of the investigation. As a control group we had 50 healthy volunteers for plasma (Monaco et al., 1975) and 10 for CSF

Address for reprint requests: Dr F. Monaco, Clinica Neurologica, Viale S. Pietro 10, 1-07100 Sassari, Italy.

Accepted 13 January 1979
(Mutani et al., 1974), along with 11 patients affected with motor neurone disease and six with hereditary ataxias.

Plasma and CSF were collected early in the morning after a 12 hour fast. We made sure that on the days immediately before examination patients were consuming a diet with a similar amount of protein, carbohydrate, and fats in order to obtain an adequate intake of all amino acids. Cerebrospinal fluid, together with serum obtained by centrifugation at $3500 \mathrm{rpm}$ for 10 minutes, was immediately deproteinised with sulphosalycilic acid after being treated with a $0.5 \%$ aqueous solution of sodium dodecylsulphate, to obtain total tryptophan recovery (Mondino et al., 1975). The determination of tryptophan and competing neutral amino acids was then performed by ion-exchange chromatography using a routine technique (Mondino, 1970).

\section{Results}

Tryptophan, leucine, isoleucine, valine, tyrosine, and phenylalanine were all diminished in the plasma of patients with multiple sclerosis (Table) but by far the greatest decrease was in the tryptophan level $(t>11)$, as well as tyrosine and phenylalanine $(t>4)$, all of them having a $P$ value of $<0.001$. A similar result was seen in the plasma of patients with motor neurone disease, but the tryptophan figures were less significant $(P<0.05)$. No difference was observed between plasma from cases of motor neurone disease and hereditary ataxias. In all diseases examined the CSF concen- 
Table Plasma and CSF tryptophan and competing neutral amino acid concentrations in patients with multiple sclerosis $(M S)$, motor neurone disease $(M N D)$, and hereditary ataxias $(H A)$ compared with normal control subjects $(N)$. $\Sigma N A A / T R Y$ is the ratio between neutral amino acids and tryptophan. Values (rounded off to full number whenever possible) are expressed in $\mu$ mol/l (mean $\pm S D$ )

\begin{tabular}{|c|c|c|c|c|c|c|c|c|}
\hline \multicolumn{5}{|l|}{ Plasma } & \multicolumn{4}{|l|}{$C S F$} \\
\hline Amino acid & $N(50)$ & $M S(32)$ & $M N D(11)$ & $H A(6)$ & $N(10)$ & $M S(32)$ & $M N D(I I)$ & $H A(6)$ \\
\hline $\begin{array}{l}\text { Tryptophan } \\
\text { Leucine } \\
\text { Isoleucine } \\
\text { Valine } \\
\text { Tyrosine } \\
\text { Phenylalanine }\end{array}$ & $\begin{aligned} 54 & \pm 9 \\
128 & \pm 27 \\
62 & \pm 15 \\
242 & \pm 48 \\
63 & \pm 13 \\
54 & \pm 12\end{aligned}$ & $\begin{array}{c}31 \pm 8^{* * *} \\
114 \pm 25^{*} \\
56 \pm 14 \\
208 \pm 49 * * \\
43 \pm 9 * * * \\
42 \pm 7^{* * *}\end{array}$ & $\begin{array}{c}26 \pm 7^{*} \\
89 \pm 26^{* * *} \\
42 \pm 12^{* * *} \\
159 \pm 42^{* * *} \\
37 \pm 8^{* * *} \\
37 \pm 9 * * *\end{array}$ & $\begin{array}{c}31 \pm 8^{* * *} \\
100 \pm 24^{*} \\
48 \pm 13^{*} \\
181 \pm 53^{* *} \\
38 \pm 12^{* * *} \\
40 \pm 22^{*}\end{array}$ & $\begin{array}{l}5 \pm 2 \\
4 \pm 2 \\
3 \pm 1 \\
9 \pm 5 \\
3 \pm 1 \\
3 \pm 2\end{array}$ & $\begin{array}{c}4 \pm 2^{*} \\
10 \pm 4^{* * *} \\
4 \pm 2 \\
17 \pm 8^{* * *} \\
5 \pm 2 \\
7 \pm 7\end{array}$ & $\begin{array}{c}2 \pm 2 * * * \\
10 \pm 6^{*} \\
3.5 \pm 2.3 \\
13 \pm 9 \\
5.5 \pm 2.8 \\
4 \pm 4\end{array}$ & $\begin{array}{c}3 \pm 1 \\
8 \pm 5 \\
2.1 \pm 2 \\
10 \pm 7 \\
5 \pm 2 \\
4 \pm 2\end{array}$ \\
\hline$\Sigma$ NAA/TRY & $9.6 \pm 3.4$ & $16 \pm 5^{*}$ & $14 \pm 5^{*}$ & $12 \pm 4^{*}$ & & & & \\
\hline
\end{tabular}

$*=\mathrm{P}<0.05 ;{ }^{* *}=\mathrm{P}<0.01 ; * * *=\mathrm{P}<0.001$ by Student's $t$ test with respect to normal subjects.

tration did not differ from normal, except for the decreased tryptophan in multiple sclerosis $(\mathrm{P}<0.05)$ and in motor neurone disease $(P<0.001)$, and the increased leucine and valine in multiple sclerosis $(\mathrm{P}<0.001)$ and in motor neurone disease $(\mathrm{P}<0.05)$.

The plasma ratio between the neutral amino acids and tryptophan ( $\Sigma$ NAA/TRY) in the different diseases gave a $P$ value of $<0.001$ for multiple sclerosis $(t>7)$ and the other degenerative diseases $(t>4)$ compared with normal subjects.

\section{Discussion}

Our results suggest that suboptimal brain tryptophan concentrations may occur in some patients with multiple sclerosis and other degenerative neurological diseases. Blood tryptophan, in fact, controls brain tryptophan concentrations and synthesis of brain 5-hydroxytryptamine (Gessa and Tagliamonte, 1974). Since brain serotonin content is regulated physiologically by plasma neutral amino acids (Fernstrom and Wurtman, 1972), the increased $\Sigma$ NAA/TRY ratio would, therefore, represent a larger brain uptake for neutral amino acids and a lower one for tryptophan. The tryptophan depletion present in patients with multiple sclerosis and other degenerative diseases might then cause a depression of the synthetic pathways for serotonin and proteins in the brain. Changes in the amount of one amino acid may, in fact, alter the synthesis and degradation of proteins in the brain or, possibly, of a specific population such as myelin proteins (Agrawal et al., 1970).

These changes are unlikely to have a primary role in the disease, but might perhaps exacerbate certain symptoms. Previous studies have demonstrated that administration of tyrosine, L-dopa, and tryptophan effectively counter the negative effect of artificial overheating on the flickerfusion threshold in patients with multiple sclerosis (Kisser and Harrer, 1974).
Finally, the different $\Sigma$ NAA/TRY ratios associated with the different neurological disorders might be used as an ancillary test in screening to differentiate between multiple sclerosis and degenerative diseases of the central nervous system.

\section{References}

Agrawal, H. C., Bone, A. H., and Davison, A. N. (1970). Effect of phenylalanine on protein synthesis in the developing rat brain. Biochemical Journal, 117, 325-331.

Fernstrom, J. D., and Wurtman, R. J. (1972). Brain serotonin content: physiological regulation by plasma neutral amino acids. Science, 178, 414-416.

Gessa, G. L., and Tagliamonte, A. (1974). Serum free tryptophan: control of brain concentrations of tryptophan and of synthesis of 5-hydroxytryptamine. In Aromatic Amino Acids in the Brain, Symposium Proceedings, pp. 206-216, Associated Scientific Publishers: Amsterdam.

Kisser, W., and Harrer, G. (1974). Amino acid determinations in serum of patients with multiple sclerosis. Wiener Klinische Wochenschrift, 86, 80-82.

Lajtha, A. (1974). Amino acid transport in the brain in vivo and in vitro. In Aromatic Amino Acids in the Brain, Symposium Proceedings, pp. 25-41. Associated Scientific Publishers: Amsterdam.

Monaco, F., Mutani, R., Durelli, L., and Delsedime, M. (1975). Free amino acids in serum of patients with epilepsy: significant increase in taurine. Epilepsia, 16, 245-249.

Mondino, A. (1970). Automatic ion-exchange chromatography of amino acids. Experimental studies for optimising resin column dimensions. Journal of Chromatography, 50, 260-273.

Mondino, A., Bongiovanni, G., and Fumero, S. (1975). A new approach for obtaining total tryptophan recovery in plasma samples deproteinized with sulphosalycilic acid. Journal of Chromatography, 104, 297-302.

Mutani, R., Monaco, F., Durelli, L., and Delsedime, M. (1974). Free amino acids in the cerebrospinal fluid of epileptic subjects. Epilepsia, 15, 593-597. 